\title{
ELECTROMAGNETIC DISSOCIATION OF Au TARGETS BY Au BEAMS IN E862
}

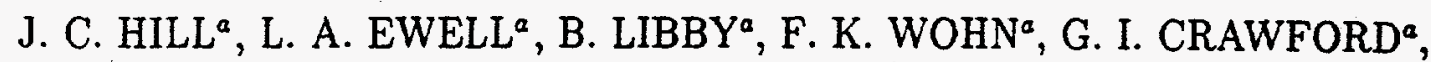 \\ W. J. TURNER ${ }^{a}$, J. B. CUMMING ${ }^{b}$, C. J. BENESH ${ }^{c}$ \\ a Dept. of Physics and Astronomy, Iowa State Univ., Ames IA 50011, USA \\ ${ }^{b}$ Chemistry Dept., Brookhaven National Lab., Upton NY 11973, USA \\ 'Physics Dept., Univ. of Northern Iowa, Cedar Falls IA 50613, USA \\ for the E866 Collaboration: \\ BNL-UCBerkeley-UCRiverside-Columbia-INS(Tokyo)-Johns \\ Hopkins-Kyoto-LLNL-Maryland-MIT-Tokyo-Tsukuba-Yonsei(Seoul)
}

\begin{abstract}
E862 is an experiment to measure Electromagnetic Dissociation (ED) of Au and Co targets by $9.89 \mathrm{GeV} /$ nucleon beams from the AGS. ED occurs at impact parameters large enough so that no strong interaction occurs, but virtual photons are exchanged, resulting in the excitation of a giant resonance. We report the first observation of an ED cross section $\left(\sigma_{E D}\right)$ larger than the total hadronic cross section for $\mathrm{Au}+\mathrm{Au}$ of 6.1 barns. Results are given for both the single- and double-neutron removal processes and compared to calculations using both semiclassical and quantum methods, including effects from both E1 and E2 giant resonances. Predictions are extended to RHIC and LHC collider energies where $\mathrm{ED}$ will be a significant determinant of the quality of the stored beams.
\end{abstract}

\section{Introduction}

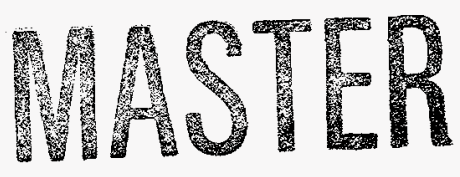

Interest in Relativistic Heavy Ion (RHI) physics centers around hadronic interactions at small impact parameters, but at the energies planned for the RHIC and LHC colliders most of the reaction cross section for heavy nuclei will be the result of non-hadronic processes due to electromagnetic interactions. E862 is an attempt to learn more about these purely electromagnetic processes that we call Electromagnetic Dissociation (ED). In RHI collisions involving impact parameters large enough so that no nuclear interaction occurs, strong electromagnetic fields are produced at the nucleus. ED occurs when a virtual photon is exchanged between nuclei. The usual result is the excitation of a giant multipole resonance. Deexcitation occurs primarily by means of neutron emission, since at giant resonance energies charged particle emission is retarded by the Coulomb barrier.

ED was first observed by Heckman and Lindstrom [1] in projectile fragmentation. Larger values were subsequently observed by our group [2] for ED of Au and Co targets. Preliminary 


\section{DISCLAIMER}

This report was prepared as an account of work sponsored by an agency of the United States Government. Neither the United States Government nor any agency thereof, nor any of their employees, make any warranty, express or implied, or assumes any legal liability or responsibility for the accuracy, completeness, or usefulness of any information, apparatus, product, or process disclosed, or represents that its use would not infringe privately owned rights. Reference herein to any specific commercial product, process, or service by trade name, trademark, manufacturer, or otherwise does not necessarily constitute or imply its endorsement, recommendation, or favoring by the United States Government or any agency thereof. The views and opinions of authors expressed herein do not necessarily state or reflect those of the United States Government or any agency thereof. 


\section{DISCLAMMER}

Portions of this document may be illegible in electronic image products. Images are produced from the best available original document. 
values of $\sigma_{E D}$ for one- and two-neutron removal from Au targets bombarded with Au beams from the AGS are given. Below we describe the experiment and the method for calculating $\sigma_{E D}$. The results are then compared with calculations using both a Weizsacker-Williams and a quantum mechanical virtual-photon spectrum considering the effects of both the E1 and E2 giant resonances. The calculations are extrapolated to the energies planned for the RHIC and LHC colliders.

\section{Experimental Method and Results}

Thin Au foil targets were bombarded with $9.89 \mathrm{GeV} /$ nucleon Au projectiles from the AGS accelerator. A low beam intensity of typically $4 \times 10^{3}$ particles per spill was used to determine the large cross section for the ${ }^{197} \mathrm{Au}\left({ }^{197} \mathrm{Au}, \mathrm{X}\right){ }^{196} \mathrm{Au}$ (one-neutron removal) reaction. This cross section was used as a secondary standard to determine the beam flux in high intensity bombardments that were used to determine the cross section for the ${ }^{197} \mathrm{Au}\left({ }^{197} \mathrm{Au}, \mathrm{X}\right){ }^{195} \mathrm{Au}$ (two-neutron removal) reaction and the nuclear correction to the above cross sections. In the low-intensity run the Au projectiles were counted using a two-element counter telescope. The saturation activities for nuclides of interest were measured by $\gamma$ counting for periods of up to several months. The intensities of the 355- and 99-keV $\gamma$ transitions were measured in order to determine the saturation activities of ${ }^{196} \mathrm{Au}$ and ${ }^{195} \mathrm{Au}$, respectively. Corrections were made for detector efficiency, coincidence summing, spread of activity in the target, production of secondaries, branching ratio and $\gamma$ absorption in the target as described earlier [3]. Total cross sections $\left(\sigma_{t o t}\right)$ were then calculated for one- and two-neutron removal.

The measured cross sections consist of the sum of an ED and a nuclear part. The nuclear contribution was determined using the concept of factorization [4]. This concept assumes that the yield of a fragment from the target due to nuclear interactions is independent of the beam except through a constant geometric factor that is the same for all fragments. Factorization has been shown [5] to be a useful concept for lighter RHI and is certainly expected to be valid for $\mathrm{Au}$ at AGS energies. The determination of the 'nuclear' contribution has a fairly large percent uncertainty but only contributes a small amount to the uncertainty of $\sigma_{E D}$ due to its dominance over the nuclear contribution. The preliminary measured results for $\sigma_{t o t}$, the nuclear contribution $\sigma_{n u c}$ and $\sigma_{E D}$ are given in Table 1.

Table 1: Cross sections for $9.89 \mathrm{GeV} /$ nucleon Au beams on Au targets.

\begin{tabular}{||c||c|c|c||}
\hline \hline Nuclide & $\sigma_{\text {tot }}(\mathrm{b})$ & $\sigma_{\text {nuc }}(\mathrm{b})$ & $\sigma_{E D}(\mathrm{~b})$ \\
\hline \hline${ }^{196} \mathrm{Au}$ & $9.28 \pm 0.53$ & $0.29 \pm 0.04$ & $8.99 \pm 0.53$ \\
\hline${ }^{195} \mathrm{Au}$ & $2.48 \pm 0.26$ & $0.16 \pm 0.02$ & $2.32 \pm 0.27$ \\
\hline
\end{tabular}




\section{Comparison of ED Cross Sections with Theory}

$\sigma_{E D}$ was calculated using the Weizsacker-Williams (WW) method [6] of virtual quanta. The cross section is obtained by integrating over the product of the equivalent photon number $N_{\gamma}$ and the photonuclear cross section $\sigma_{\gamma}$. For Au the $\sigma_{\gamma}$ used was that of Veyssiere et al. [7] but multiplied by a factor of 0.93 [8]. At ultrarelativistic energies $N_{\gamma}$ is the same for different multipolarities so that the experimental $\sigma_{\gamma}$ can be used, but at intermediate energies $N_{\gamma}$ is different for different [9] multipolarities. It is thus necessary to to break up $\sigma_{E D}$ into different components corresponding to different multipolarities.

Only the E1 and E2 giant resonances make significant contributions to the cross sections. Corrections for the effect of both the isoscalar and isovector $\mathrm{E} 2$ resonances are made using the method of Norbury [10]. The E2 cross sections were determined using a Lorentzian parameterization. The resonance energies and widths used were 10.8 and $2.9 \mathrm{MeV}$ for the isoscalar and 23.0 and $7.0 \mathrm{MeV}$ for the isovector $\mathrm{E} 2$ resonances, respectively, as given by Bertrand $e t$ al. [11]. In both cases a sum rule strength of 95 percent was assumed. Various prescriptions for the minimum impact parameter have been used. Here the parameterization of Benesh, Cook and Vary [12] is used, as in our earlier work. An alternative parameterization by Kox et al. [13] gives slightly smaller values for $\sigma_{E D}$.

The measured $\sigma_{E D}$ for one- and two-neutron removal from Au targets by Au beams from the AGS is compared in Fig. 1 with the WW calculations described above. Measurements performed by Aumann et al. [14] using Au beams with energies of $1.0 \mathrm{GeV} /$ nucleon are also shown for comparison. The calculations are in approximate agreement wi h the measured values but systeme:ic deviations are evident. The calculations consistently overestimate the one-neutron removal cross sections but underestimate the two-neutron removal cross sections.

$\sigma_{E D}$ has been recalculated using a procedure of Benesh et al. [15]. The basic folding method of the WW procedure was recovered as well as the effects of both the E1 and E2 resonances, but a fully quantum-mechanical expression for the 'equivalent-photon spectrum' was used. The effect of the 'quantum' virtual photon spectrum is that the predicted cross sections are lower, producing very good agreement for the one-neutron removal cross section but poorer agreement for the two-neutron removal cross section.

It has been suggested by Llope and Braun-Munzinger [16] that it is possible to absorb several photons in a collision, leading to the excitation of multiphonon giant E1 resonance states. Aumann et al. [14] estimated the effect for $1.0 \mathrm{GeV} /$ nucleon $\mathrm{Au}$ beams on $\mathrm{Au}$ targets by including the effect of the excitation of the two phonon giant E1 resonance. They predicted an enhancement of the two- and three-neutron removal cross sections at the expense of the one-neutron removal cross section but were not able to obtain complete agreement. This could be the explanation for the excess observed two-neutron removal cross section.

\section{Extrapolation to Collider Energies}

The ED process will be a major determinant of the quality and lifetime of stored relativistic heavy ion beams in the next generation of colliders at RHIC and the LHC due to the rapid rise in $\sigma_{E D}$ as beam energies are increased. Calculations of cross sections for both 


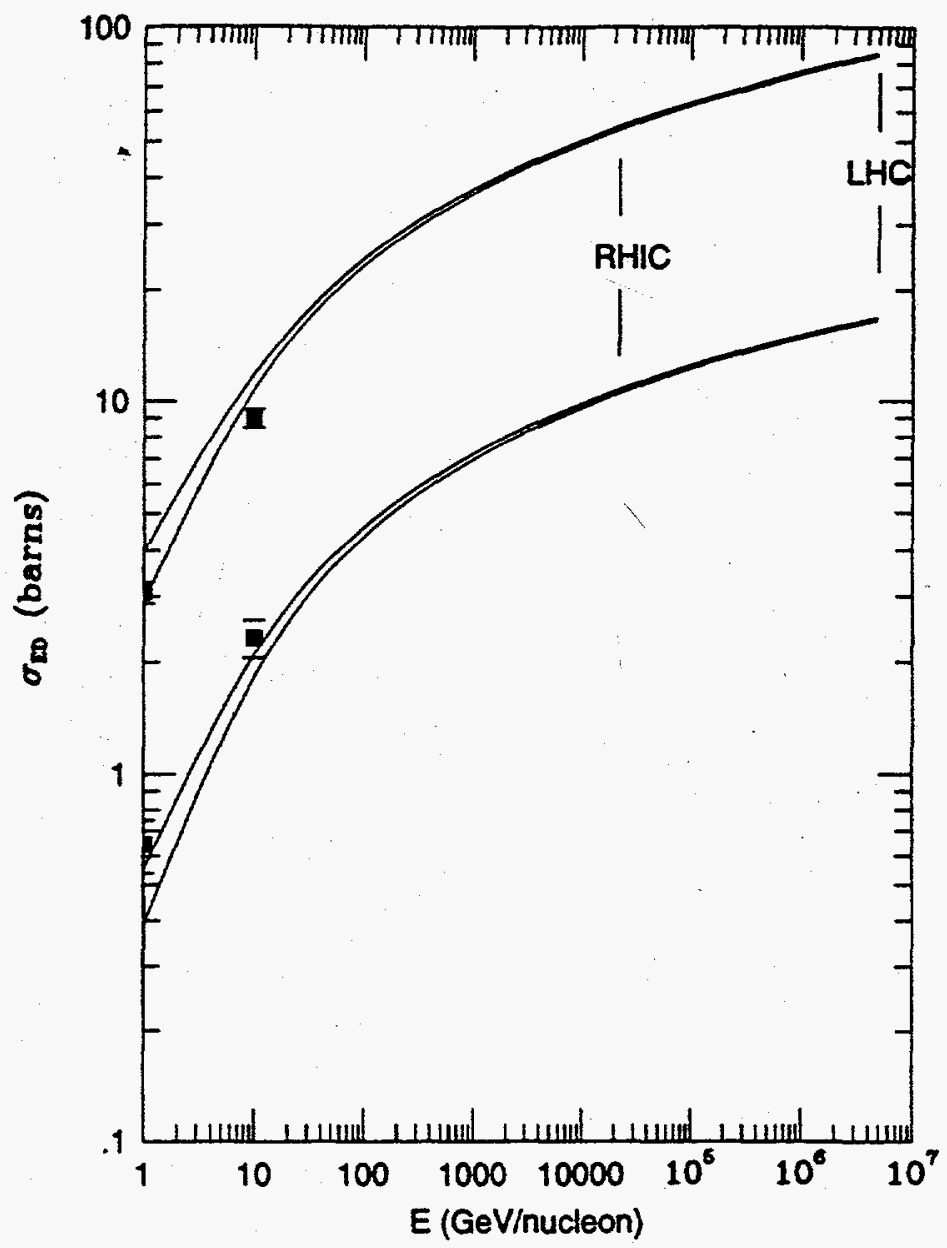

Figure 1: Calculated $\sigma_{E D}$ for Au beams incident on a Au target. The upper and lower curves are for oneand two-neutron removal reactions respectively. Results using 'quantum' $N_{\gamma}$ are slightly lower than those using WW $N_{\gamma}$. Experimental results from [14] at $1 \mathrm{GeV} /$ nucleon and this work at AGS energies are shown.

the one- and two-neutron removal ED reactions have thus been extended to include values expected at collider energies. The results are shown in Fig. 1. For both curves the effects of the E2 resonances have been included. The results of calculations using both the WW and the 'quantum' $N_{\gamma}$ are shown. The x axis shows the energy for a projectile incident on a stationary target. The equivalent collider energies for $\mathrm{Au}$ on $\mathrm{Au}$ at RHIC and the LHC are indicated on the figure. Note that at collider energies the two calculations give approximately the same results.

For $100 \mathrm{GeV} /$ nucleon colliding Au beams at RHIC the one- and two- neutron removal $\sigma_{E D}$ s are estimated to be about 52 and 11 barns, respectively. For the $4 \mathrm{TeV} /$ nucleon beams expected for the LHC the corresponding $\sigma_{E D}$ s are about 85 and 16 barns, respectively. For $\mathrm{Pb}$ on $\mathrm{Pb}$ at the LHC at the same energy per nucleon, the cross sections would be expected to be about 10 percent higher. 


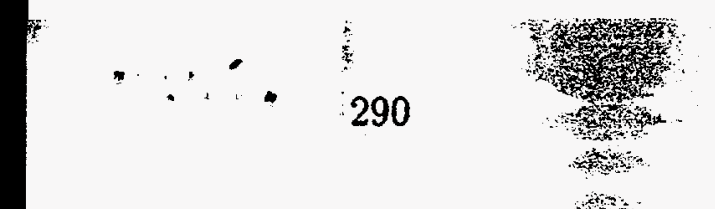

\section{Conclusions}

$\sigma_{E D}$ was measured for the one- and two-neutron removal reactions from AGS Au beams on $\mathrm{Au}$ targets. The preliminary cross sections were $8.99 \pm 0.53$ and $2.32 \pm 0.27$ barns, respectively, as compared to the total hadronic cross section (geometric) of 6.12 barns. Calculations using a simple WW approach slightly overestimates the one-neutron removal and underestimates the two-neutron removal cross section. Consideration of the contribution of the E2 resonance is necessary at these intermediate energies but agreement with experiment is still not excellent. Good agreement is obtained for the one-neutron removal cross section by the use of a fully quantum-mechanical expression for the virtual-photon spectrum. Inclusion of multiple photon excitations leading to multiphonon giant resonance states may need to be considered to obtain better agreement with the two-neutron removal cross sections. Extrapolation of the Au+Au one-neutron removal cross sections gives about 52 barns and 82 barns for energies expected for the RHIC and LHC colliders, respectively.

\section{Acknowledgements}

The authors thank D. Beavis for help during bombardments at the AGS. Experiment E862 is supported by the U.S. Department of Energy Under Contract No. DE-FG0292ER40692.

\section{References}

1. H.H. Heckman and P.J. Lindstrom, Phys. Rev. Lett. 37, 56 (1976)

2. M.T. Mercier et al., Phys. Rev. C33, 1655 (1986)

3. J.C. Hill et al., Phys. Rev. C38, 1722 (1988)

4. D.L. Olson et al., Phys. Rev. C28, 1602 (1983)

5. S.B. Kaufman et al., Phys. Rev. C22, 1897 (1980)

6. E.J. Williams, Proc. Roy. Soc. A139, 163 (1933)

7. A. Veyssiere et al., Nucl. Phys. A159, 561 (1970)

8. B.L. Berman et al., Phys. Rev. C36, 1286 (1987)

9. C.A. Bertulani and G. Baur, Phys. Rep. 163, 299 (1988)

10. J.W. Norbury, Phys. Rev. C41, 3721990

11. F.E. Bertrand, Annu. Rev. Nucl. Sci. 26, 457 (1976)

12. C.J. Benesh et al., Phys. Rev. C40, 1198 (1989)

13. S. Kox et al., Phys. Rev. C35, 1678 (1987)

14. T. Aumann et al., Phys. Rev. C47, 1728 (1993)

15. C.J. Benesh et al., Phys. Rev. C54, 1404 (1996)

16. W.J. Llope and P. Braun-Munzinger, Phys. Rev. C41, 2644 (1990) 